\title{
Effectiveness and Safety Evaluation of Thrice Weekly Double Strength vs Daily Single Strength Trimethoprim-Sulfamethoxazole for Prophylaxis of Pneumocystis Jirovecii Pneumonia after Kidney Transplantation: A Two-Year Prospective Cohort Study
}

\author{
Hui Yang ${ }^{1}$, Lizhen Pang ${ }^{2}$, Xiaopeng $\mathrm{Hu}^{3}$, Wei Wang ${ }^{3}$, Bifang $\mathrm{Xu}^{2}$, Xiaodong Zhang ${ }^{3}$, and Lihong $\mathrm{Liu}^{1}$ \\ ${ }^{1}$ Department of Pharmacy, Beijing Chao-Yang Hospital, Capital Medical University, Beijing, China; ${ }^{2}$ Department of \\ Pharmacy, Cancer Hospital of HuanXing Chao Yang District Beijing, Beijing, China; \\ ${ }^{3}$ Department of Urology, Beijing Chao-Yang Hospital, Capital Medical University, Beijing, China
}

\begin{abstract}
Corresponding Authors: Xiaodong Zhang, Urology Department of Beijing Chao-Yang Hospital, 8 Gongren Tiyuchang Nanlu, Chaoyang District, Beijing, China; TEL: (+86) 010 85231747; email: zhangxiaodong@bjicyh.com; and Lihong Liu, Pharmacy Department of Beijing Chao-Yang Hospital, 8 Gongren Tiyuchang Nanlu, Chaoyang District, Beijing, China; TEL: (+86) 10 85231077; FAX: (+86) 10 85231077, email: hongllh@126.com.
\end{abstract}

Received, November 5, 2020; Revised, April 12, 2021; Accepted, April 13, 2021; Published, May 4, 2021

\begin{abstract}
Purpose: It is hard for clinicians to choose the best regimen for pneumocystis jirovecii pneumonia (PJP) prophylaxis. The aim is to evaluate the effectiveness and safety of thrice weekly double strength (TWDS) vs daily single strength (DSS) trimethoprim-sulfamethoxazole (TMP-SMX) for prophylaxis of PJP after kidney transplantation. Methods: Adult renal transplant recipients (RTRs) who were transplanted between January 1, 2015 and July 1, 2018 were evaluated. A total of 189 RTRs were prescribed PJP prophylactic regimen during the study period (TWDS group: $n=98$; DSS group: $n=91$ ). Results: Morbidity due to PJP infection was significantly higher in TWDS group as compared with DSS group $(8.60 \%$ vs $1.14 \%$, $\mathrm{p}=0.021)$. There was a significant trend toward higher prevalence of confirmed PJP $(\log -\operatorname{rank}=0.021)$ in TWDS group. The use of DDS TMP-SMX for prophylaxis after kidney transplantation was associated with a $79 \%$ reduction in the incidence of PJP comparing the prophylactic regimen of TWDS. There was no significant difference between the two groups in the overall rate of premature TMP-SMX discontinuation and laboratory indexes. Conclusion: Six months of DSS TMP-SMX prophylaxis was more effective than TWDS TMP-SMX regimen with the same safety profile.
\end{abstract}

\section{INTRODUCTION}

Pneumocystis jiroveci, formerly pneumocystis carinii, remains an important opportunistic fungal pathogen in renal transplant recipients (RTRs) (1). In the absence of effective prophylaxis, $5 \%-15 \%$ solid organ transplant recipients may develop pneumocystis jirovecii pneumonia (PJP) posttransplant (2-3). Thus, the updated guidelines from the Infectious Diseases Community of Practice of the American Society of Transplantation recommends anti-pneumocystis prophylaxis for all solid organ transplant recipients (4). Trimethoprimsulfamethoxazole (TMP-SMX) is the first-line agent for prevention of PJP, all other prophylactic agents (i.e., dapsone, atovaquone, pentamidine) should be considered second line agents due to drug intolerance, cost, and effectiveness issues.

TMP-SMX associates with numerous adverse drug reaction, including leukopenia, absolute neutropenia, thrombocytopenia, anemia, and hepatitis. Besides that, trimethoprim has the capacity to inhibit potassium and creatinine secretion resulting in hyperkalemia and elevation of serum creatinine (4). Post-transplant management, therefore, becomes a balancing act between preventing PJP on the one hand and managing the adverse effects of TMP-SMX on the other. Despite consensus about the significant role of TMP-SMX in routine post-transplant care, there remains a remarkable vagueness about how TMP-SMX should best be used for PJP prophylaxis as the dose, frequency and duration are inconsistent. Three guidelines suggested that TMP-SMX $80 \mathrm{mg} / 400 \mathrm{mg}$ (single strength) daily or $160 \mathrm{mg} / 800 \mathrm{mg}$ (double strength) three times weekly (4-6). And the British Transplantation Society only suggested that TMP SMX $80 \mathrm{mg} / 400 \mathrm{mg}$ daily for PJP prophylaxis. The duration of PJP prophylaxis had been prolonged in recent years. Guidelines published before 2017 suggested that the duration of PJP prophylaxis is at least 4 months or $3-6$ months post-transplant (6-9). Guidelines published after 2017 suggested that the 
duration of the PJP prophylaxis treatment should be $6-12$ months (4-5).

None of the guidelines are evidence-based and leave the decision to clinicians to prefer one dosing regimen over the other. Thus, the aim of this study is to evaluate the effectiveness and safety of thrice weekly double strength (TWDS) vs daily single strength (DSS) TMP-SMX for prophylaxis of PJP after kidney transplantation.

\section{METHODS}

\section{Study design and patient population}

This study was conducted over a two-year period at Chaoyang hospital in Beijing, China This was a prospective cohort study evaluating the effectiveness and safety of two strategies for prophylaxis of PJP (TWDS vs DSS) (Ethics Approval: 2019-7-2-1). Sodium bicarbonate has the capacity to promote TMP-SMX secretion reducing the risk of kidney injury according to the package insert. Hence, two tablets of sodium bicarbonate with one tablet of TMP-SMX were given to the patients at the same time. Adult renal transplant recipients (RTRs) transplanted between January 1, 2015 and July 1, 2018 were evaluated. Patients who received TWDS or DSS TMP-SMX for prevention of PJP for about 6 months post-transplant were eligible. All the patients were given either an interleukin-2 receptor antagonist or rabbit ant-thymocyte globulin (rATG) to ensure similar induction regimens. Tacrolimus (TAC), mycophenolate mofetil (MMF) and glucocorticoids were standard initial treatments to maintain immunosuppressive strategy and prevent allograft rejection in our center. Post-transplant laboratory monitoring is performed weekly for the first three months, once every two weeks for the next three months, and then monthly thereafter. Reasons for exclusion from this analysis included 1) patients received a multi-organ transplant, 2) the glomerular filtration rate of the patients were less than $30 \mathrm{~mL} / \mathrm{min}$ on discharge day, 3) patients did not follow-up.

\section{Data collection}

Inpatient/outpatient physical and electronic medical records, including laboratory data, clinic visit notes, and medication histories, were reviewed for demographics, laboratory values, immunosuppressive therapies, transplant characteristics, pathology reports, and allograft outcomes.

\section{Outcomes}

The effectiveness outcome was incidence of PJP (the diagnosis of PJP was confirmed based on microscopy with staining or a polymerase chain reaction). The safety outcomes were the early discontinuation rate of TMP-SMX and the incidence of laboratory abnormalities (hyperkalemia, defined as serum potassium $\geqslant 5.5 \mathrm{mmol} / \mathrm{L}$; total leukopenia, defined as total $\mathrm{WBC} \leqslant 3000 / \mathrm{cu} \mathrm{mm}$; absolute neutropenia, defined as neutrophil count $\leqslant$ 500/cumm; thrombocytopenia, defined as platelet count $\leqslant 100,000 / \mathrm{cu} \mathrm{mm}$; anemia, defined as hemoglobin $\leqslant 100 \mathrm{~g} / \mathrm{L}$. and hepatitis, defined as alkaline phosphatase $\geqslant 125 \mathrm{IU} / \mathrm{mL}$, alanine aminotransferase $\geqslant 45 \mathrm{IU} / \mathrm{mL}$, and/or aspartate aminotransferase $\geqslant 40 \mathrm{IU} / \mathrm{mL}$ ).

\section{Statistical analysis}

Standard descriptive statistics were used to summarize the study population characteristics. The Fisher's exact or chi - square tests for categorical variables and Student's t-test for continuous variables were used. Continuous variables were presented as the mean, with the standard deviation (SD). The overall morbidity was analyzed using KM curves. The log - rank test was used to compare incidence of PJP between two groups throughout a period of 24 months. The y-axis represents the cumulative incidences of PJP between those two groups, with percentage as its unit. And the $\mathrm{x}$-axis represents the time period we followed up, with month as its unit. A two - sided test was performed and a $\mathrm{P}$ - value $<0.05$ was considered to be statistically significant. Univariable Cox proportional hazards regression was used to evaluate variables as a potentially protective factor for incidence of PJP. Data were analyzed using SPSS 25 statistical software.

\section{RESULTS}

A total of 189 RTRs were prescribed PJP prophylactic regimen during the study period (TWDS group: $\mathrm{n}=98$; DSS group: $\mathrm{n}=91$ ). Baseline demographics of the two groups were comparable (Table 1, $\mathrm{p}>0.05$ ). Duration of prophylaxis was the same between two groups $(5.08 \pm 1.85$ vs $5.75 \pm$ $3.04, \mathrm{p}=0.073)$. For the risk factors for PJP, there was no significant difference in 1-year and 2-year allograft rejection rate, but 1-year CMV infection rate was higher in the DSS group than the TWDS group $(15.31 \%$ vs $32.97 \%, p=0.04$, Table 1$)$.

\section{Incidence of PJP}

The overall morbidity of PJP infection in the TWDS group was higher than that in the DSS group $(8.60 \%$ vs $1.14 \%, \mathrm{p}=0.021)$. The 1 -year incidence of PJP infection after transplantation was $7.53 \%$ in the TWDS group and $1.14 \%$ in the DSS group $(\mathrm{P}=$ 0.037), and the incidence of PJP infection occurring 
Table 1. Baseline characteristics of total study subjects

\begin{tabular}{|c|c|c|c|}
\hline Characteristic & $\begin{array}{l}\text { TWDS group } \\
(\mathrm{n}=98)\end{array}$ & $\begin{array}{l}\text { DSS group } \\
(\mathrm{n}=91)\end{array}$ & $P$ value \\
\hline $\operatorname{Age}(y)$ & $41.38 \pm 10.72$ & $41.80 \pm 11.47$ & 0.79 \\
\hline Height $(\mathrm{cm})$ & $168.83 \pm 14.07$ & $166.76 \pm 13.65$ & 0.31 \\
\hline Weight(kg) & $66.35 \pm 15.39$ & $64.34 \pm 16.90$ & 0.31 \\
\hline Gender,male(n,\%) & $70(71.43)$ & $54(59.34)$ & 0.08 \\
\hline Han people(n,\%) & $95(96.94)$ & $90(98.90)$ & 0.35 \\
\hline Smoking $(\mathrm{n}, \%)$ & $21(21.43)$ & $16(17.58)$ & 0.51 \\
\hline Alcohol consumption(n, $\%)$ & $10(10.20)$ & $10(1.10)$ & 0.86 \\
\hline \multicolumn{4}{|l|}{ Comorbidity } \\
\hline Hypertension(n,\%) & $83(84.69)$ & $78(85.71)$ & 0.84 \\
\hline Diabetes(n,\%) & $10(10.20)$ & $5(5.49)$ & 0.23 \\
\hline hyperlipemia(n,\%) & $1(1.02)$ & $3(3.30)$ & 0.28 \\
\hline hyperuricemia(n,\%) & $4(4.08)$ & $3(3.30)$ & 0.78 \\
\hline $\mathrm{CHD}(\mathrm{n}, \%)$ & $0(0.00)$ & $2(2.20)$ & 0.14 \\
\hline \multicolumn{4}{|l|}{ Laboratory data } \\
\hline Creatinine(umol/L) & $860.60 \pm 266.94$ & $831.83 \pm 270.34$ & 0.46 \\
\hline Urea nitrogen $(\mathrm{mmol} / \mathrm{L})$ & $21.43 \pm 9.10$ & $21.74 \pm 8.06$ & 0.81 \\
\hline $\mathrm{TC}(\mathrm{mmol} / \mathrm{L})$ & $4.33 \pm 1.82$ & $4.45 \pm 1.03$ & 0.58 \\
\hline LDL-C(mmol/L) & $2.42 \pm 1.91$ & $2.25 \pm 0.75$ & 0.42 \\
\hline Leucocyte $\left(* 10^{\wedge} 9 / \mathrm{L}\right)$ & $7.22 \pm 2.71$ & $6.94 \pm 2.06$ & 0.43 \\
\hline Neutrophilic(\%) & $71.64 \pm 10.52$ & $71.09 \pm 10.58$ & 0.72 \\
\hline Lymphocytes $(\%)$ & $19.52 \pm 8.97$ & $19.75 \pm 8.09$ & 0.85 \\
\hline Hemoglobin $(g / L)$ & $112.38 \pm 19.45$ & $113.90 \pm 21.16$ & 0.61 \\
\hline \multicolumn{4}{|l|}{$\begin{array}{l}\text { Induction drug after kidney } \\
\text { transplantation }\end{array}$} \\
\hline ATJ(ramus) & $5.09 \pm 2.63$ & $4.76 \pm 2.04$ & 0.50 \\
\hline Basiliximab(ramus) & $1.92 \pm 0.28$ & $1.93 \pm 0.26$ & 0.79 \\
\hline \multicolumn{4}{|l|}{ Etiology of ESRD } \\
\hline Glomerulonephritis (n,\%) & $27(27.55)$ & $26(28.57)$ & 0.88 \\
\hline Polycystic kidney (n,\%) & $4(4.08)$ & $7(7.69)$ & 0.29 \\
\hline $\begin{array}{l}\text { Hypertensive nephropathy } \\
(\mathrm{n}, \%)\end{array}$ & $4(4.08)$ & $5(5.49)$ & 0.65 \\
\hline Tubal nephritis (n,\%) & $2(2.04)$ & $0(0)$ & 0.17 \\
\hline Diabetic nephropathy (n,\%) & $3(3.06)$ & $3(3.30)$ & 0.93 \\
\hline Other causes(n,\%) & $4(4.08)$ & $7(7.69)$ & 0.29 \\
\hline Unclear(n,\%) & $54(55.10)$ & $43(47.25)$ & 0.28 \\
\hline \multicolumn{4}{|l|}{ Risk factors for PJP } \\
\hline $\begin{array}{l}1 \text {-year CMV infection rate } \\
(\mathrm{n}, \%)\end{array}$ & $15(15.31)$ & $30(32.97)$ & 0.04 \\
\hline $\begin{array}{l}\text { 2-year CMV infection rate } \\
\text { (n, \%) }\end{array}$ & $1(1.02)$ & $1(1.10)$ & 0.96 \\
\hline 1 -year AR rate $(\mathrm{n}, \%)$ & $1(1.02)$ & $1(1.10)$ & 0.96 \\
\hline 2-year AR rate (n, \%) & $0(0.00)$ & $0(0.00)$ & l \\
\hline
\end{tabular}

TWDS, thrice weekly double strength; DSS, daily single strength; LDL-C=low density lipoprotein cholesterol, TC $=$ total cholesterol, $\mathrm{ATJ}=$ Antithymocyte globulin, $\mathrm{CHD}=$ coronary artery heart disease, $\mathrm{ESRD}=$ end-stage renal disease, $\mathrm{CMV}=$ cytomegalovirus, $\mathrm{AR}=$ allograft rejection.

within the 12 months to 24 months after transplantation was $1.08 \%$ and $0.00 \%$, respectively $(\mathrm{p}=0.329)$. For patients withPJP, $47.06 \%$ have risk factors for pneumocystis pneumonia $(30.77 \%$ vs $100 \%, \mathrm{p}=0.029)$. The Kaplan-Meier curve showing time to development of PJP is in Figure 1. There was a significant trend toward higher prevalence of confirmed PJP in the TWDS group versus the DSS group by 24 months after transplantation (log rank=0.021). The use of DDS TMP-SMX for 
prophylaxis after kidney transplantation was associated with a $79 \%$ reduction in the incidence of PJP (hazard ratio $[\mathrm{HR}]=0.21 ; 95 \% \mathrm{CI}=0.06-0.79$ ) comparing the prophylactic regimen of TWDS.

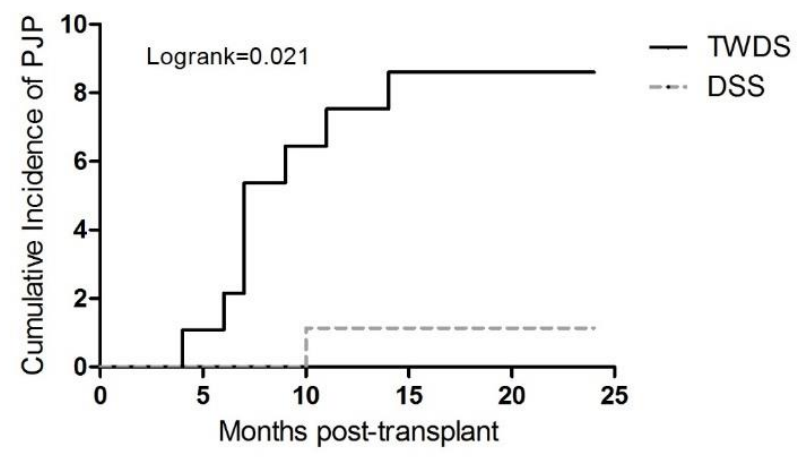

Figure 1. Cumulative incidence of pjp receiving TWDS or DSS.

\section{Early discontinuation of TMP-SMX}

Of the 8 patients who discontinued TMP-SMX early, 7 had documented reasons. Three patients experienced elevation of serum creatinine, one patient experienced leukopenia, the remaining 3 stopped taking the drugs themselves. The overall rate of premature TMP-SMX discontinuation was similar between the groups $(5.1 \%$ vs $3.3 \% ; \mathrm{P}=0.538$, Table 2).

\section{Laboratory abnormalities}

There was no significant difference between the two groups in the laboratory indexes before taking TMPSMX.. After taking TMP-SMX, no significant difference was found between the two groups in any laboratory indexes except the incidence of hyperkalemia $(5.128 \%$ vs $0.00 \%, \mathrm{p}=0.032$, Table 3$)$.

\section{DISCUSSION}

This was a prospective study evaluating the impact of two different 6-month prophylactic regimens of TMP-SMX, TWDS versus DSS, on the prevalence of PJP disease in 189 RTRs. Our findings suggested that six months of DSS TMP-SMX prophylaxis was more effective than TWDS TMP-SMX regimen with the same safety profile. And for all we know, this was the first clinical trial comparing TWDS to DSS TMP-SMX for six months in de-novo KTR during the past 30 years.

Routine anti-pneumocystis prophylaxis is generally recommended in most transplant centers. Our study demonstrated that six months of DSS TMP-SMX regimen was more effective than TWDS TMP-SMX regimen for PJP prophylaxis in RTRs. Our result was different from previous studies published in the 1980s (10-12). Three studies published in the 1980s found no difference in the rate of PJP infections after daily vs thrice-weekly prophylaxis. It might be due to different regimen of immunosuppressive agents. Increased understanding of immune rejection has led to the development of safe modern immunosuppressive agents. Along with the low incidence of post-transplant rejection, nonspecific side effects of immunosuppression include infection, particularly opportunistic infections increased (13). So more effective and safety prophylactic regimens for PJP is needed.

Now with widespread use of prophylaxis and changing immunosuppressant regimens, the incidence post-transplant is uncertain but appears to range from 0.3 to $2.5 \%$ (14-17). The overall morbidity of PJP infection in our studies was higher $(4.97 \%)$ than previous published study. It might be due to the immunosuppressive regimen selected. Nowadays, induction therapy (either basiliximab or antithymocyte globulin) is widely used, and studies had demonstrated that induction therapy was associated with an increased risk of infection (18-19). It led to the increase of the morbidity of PJP infection. In addition, recent reports have shown that despite effective prophylaxis for 6-12 months posttransplantation, PJP may emerge (20). Risk factors include cytomegalovirus infection (CMV) and graft rejection (14). Most patients with PJP had risk factors for pneumocystis pneumonia in our study (30.77\% and $100 \%$, respectively). CMV infection rate was higher in DSS group than TWDS group. It might be due to increasing concerns about CMV infection. However, even the risk to get PJP infection was higher in DSS group, our study demonstrated that the use of DDS TMP-SMX for prophylaxis after kidney transplantation was associated with a $79 \%$ reduction in the incidence of PJP comparing the prophylactic regimen of TWDS.

The prophylactic duration had been prolonged in recent years. Guidelines recommended 6-12 months of PJP prophylaxis after transplantation, SOT patients remain at risk for PJP beyond complete course of prophylaxis (21). Goto et al recommended lifelong prophylaxis of kidney transplant recipients to prevent new outbreaks (22). Most patients suffering from PJP have risk factors of pneumocystis pneumonia. Extended prophylaxis targeting recipients with risk factors may reduce the incidence of PJP. Targeted prophylaxis may be more feasible.

Our study demonstrated that both TWDS and DSS were well-tolerated regimens for PJP prophylaxis in RTRs. The incidence of hyperkalemia was lower in the DSS group compared to the TWDS group in the first week after taking TMP-SMX. Then no significant difference between the two groups was found in the laboratory abnormalities. Only eight patients (4.23\%) discontinued TMP-SMX before the 
prescribed 6-month posttransplant mark in total in our study. Other published studies reported poor tolerability, as evidenced by higher discontinuation rates. Giullian et al used a dose of 1 single-strength tablet daily of TMP-SMX and reported a discontinuation rate of $40 \%$. But the main reason for stopping TMP-SMX prematurely was drug hypersensitivity reaction (allergy, rash, or noninfectious fever) ( $\mathrm{n}=16,25 \%)$ (23). Sulfonamides is an essential drug for urinary tract infections in China, therefore most of the patients used TMP-SMX before. The doctors would not prescribe TMP-SMX for the patients who had a TMP-SMX allergy history. Hence, avoidance of antibiotic sulfonamides in patients with a history of a sulfonamide allergy is largely necessary, and this will decrease the discontinuation rate to a great extent. Mitsides and colleagues reported a discontinuation rate of $38 \%$ when using the same dose of 1 single-strength tablet of TMP-SMX once daily. Reported reasons for discontinuation included acute kidney injury, gastrointestinal symptoms, and leukopenia (24). In our prophylactic regimen, the patients were given one tablets of TMP-SMX with two tablets of sodium bicarbonate at the same time. Sodium bicarbonate has the capacity to promote TMP-SMX secretion reducing the risk of kidney injury according to the package insert. Besides that, asking the patients to drink more water after taking TMP-SMX is another effective way for risk reduction.

Limitations of this study include the fact that the data were collected at a single center. Both the DSS group and TWDS group had fewer patients for comparison. Second, pneumocystis colonization can also be a challenging problem in transplant recipients despite the use of TMP-SMX prophylaxis.

\section{CONCLUSION}

In summary, we demonstrated that six months of DSS TMP-SMX prophylaxis was more effective than a TWDS TMP-SMX regimen with the same safety profile. Further randomized controlled trial comparing DSS to TWDS TMP-SMX in RTRs is justified.

Table 2. Early discontinuation of TMP-SMX

\begin{tabular}{llllll}
\hline & $\begin{array}{l}\text { Elevation of serum } \\
\text { creatinine }(\mathrm{n}, \%)\end{array}$ & $\begin{array}{l}\text { Leukopenia } \\
(\mathrm{n}, \%)\end{array}$ & $\begin{array}{l}\text { Self-discontinuation } \\
(\mathrm{n}, \%)\end{array}$ & $\begin{array}{l}\text { Unknown } \\
(\mathrm{n}, \%)\end{array}$ & $\begin{array}{l}\text { Total } \\
(\mathrm{n}, \%)\end{array}$ \\
\hline $\begin{array}{l}\text { TWDS group } \\
(\mathrm{n}=98)\end{array}$ & 1 & 1 & 3 & 0 & 5 \\
$\begin{array}{l}\text { DSS group } \\
(\mathrm{n}=91)\end{array}$ & $(1.02)$ & $(1.02)$ & $(3.06)$ & $(0.00)$ & $(5.10)$ \\
$\mathrm{P}$ & $(2.20)$ & 0 & 0 & 1 & 3 \\
\hline
\end{tabular}

TWDS, thrice weekly double strength; DSS, daily single strength.

Table 3. Laboratory abnormalities after taking TMP-SMX

\begin{tabular}{llll}
\hline Characteristics & TWDS group & DSS group & P value \\
\hline One day before taking TMP-SMX & & & \\
Serum level of creatinine (umol/L) & $99.36 \pm 34.91$ & $107.30 \pm 31.47$ & 0.13 \\
Hyperkalemia (n,\%) & $0(0.00)$ & $0(0.00)$ & $/$ \\
Total leukopenia (n,\%) & $0(0.00)$ & $2(2.27)$ & 0.18 \\
Absolute neutropenia (n,\%) & $15(19.23)$ & $21(23.86)$ & 0.47 \\
Thrombocytopenia (n,\%) & $3(3.85)$ & $3(3.41)$ & 0.88 \\
Anemia (n,\%) & $43(55.13)$ & $60(68.18)$ & 0.08 \\
ALT or AST (n,\%) & $11(14.86)$ & $13(16.05)$ & 0.84 \\
One week after taking TMP-SMX & & & \\
Serum level of creatinine (umol/L) & $107.80 \pm 35.13$ & $113.28 \pm 31.95$ & 0.29 \\
Hyperkalemia (n,\%) & $4(5.13)$ & $0(0.00)$ & 0.03 \\
Total leukopenia (n,\%) & $0(0.00)$ & $2(2.27)$ & 0.18 \\
Absolute neutropenia (n,\%) & $15(19.23)$ & $23(26.14)$ & 0.29 \\
Thrombocytopenia (n,\%) & $1(1.28)$ & $2(2.27)$ & 0.63 \\
Anemia (n,\%) $\%$ ) & $17(21.79)$ & $31(35.23)$ & 0.06 \\
ALT or AST (n,\%) & $13(16.67)$ & $15(17.05)$ & 0.95
\end{tabular}

Table 3 continues... 


$\begin{array}{llll}\text { One month after taking TMP-SMX } & & & \\ \text { Serum level of creatinine (umol/L) } & 104.39 \pm 31.93 & 113.15 \pm 32.41 & 0.08 \\ \text { Hyperkalemia (n,\%) } & 1(1.27) & 2(2.30) & 0.62 \\ \text { Total leukopenia (n,\%) } & 1(1.27) & 1(1.27) & 0.95 \\ \text { Absolute neutropenia (n,\%) } & 35(44.30) & 37(42.53) & 0.82 \\ \text { Thrombocytopenia (n,\%) } & 1(1.27) & 4(4.60) & 0.21 \\ \text { Anemia (n,\%) } & 8(10.13) & 15(17.24) & 0.19 \\ \text { ALT or AST (n,\%) } & 3(3.40) & 7(8.05) & 0.25 \\ \text { Six month after taking TMP-SMX } & & & \\ \text { Serum level of creatinine (umol/L) } & 103.56 \pm 29.91 & 108.16 \pm 24.86 & 0.30 \\ \text { Hyperkalemia (n,\%) } & 1(1.28) & 0(0.00) & 0.29 \\ \text { Total leukopenia (n,\%) } & 2(2.56) & 4(5.19) & 0.50 \\ \text { Absolute neutropenia (n,\%) } & 42(53.85) & 48(63.16) & 0.24 \\ \text { Thrombocytopenia (n,\%) } & 3(3.85) & 1(1.32) & 0.26 \\ \text { Anemia (n,\%) } & 2(2.56) & 4(5.26) & 0.50 \\ \text { ALT or AST (n,\%) } & 6(7.69) & 7(9.10) & 0.75\end{array}$

TWDS, thrice weekly double strength; DSS, daily single strength; TMP-SMX, trimethoprim-sulfamethoxazole; ALT, alanine aminotransferase; AST, aspartate aminotransferase.

CONFLICT OF INTEREST. The authors declare no conflicts of interest.

FUNDING DISCLOSURE. This research did not receive any specific grant from funding agencies in the public, commercial, or not-for-profit sectors.

ETHICAL APPROVAL. This study was approved by the institutional review board of Hospital. (Ethics Approval: 2019-7-2-1). This work was followed the Declarations of Helsinki and Declaration of Istanbul guidelines and that no executed prisoner was used as a donor. The table indicating the medical explanations for brain death of the donors in our study was provided in Supporting Table 1.

CONTRIBUTORS. HY and LZP contributed equally to this work. HY, and LHL designed the experiments. HY, LZP, XPH, WW, XDZ and BFX collected and analyzed the data. This article was written by HY.

\section{REFERENCES}

1. Martin SI, Fishman JA and The AST infectious diseases Community of Practice. Pneumocystis pneumonia in solid organ transplantation. Am J Transplant. 2013; 13(Suppl 4): 272-9. doi: 10.1111/ajt.12119.

2. Iriart $\mathrm{X}$, Bouar $\mathrm{ML}$, Kamar $\mathrm{N}$, et al. Pneumocystis pneumonia in solid - organ transplant recipients. J Fungi (Basel). 2015; 1(3): 293-331. doi: 10.3390/jof1030293.

3. De Castro $\mathrm{N}, \mathrm{Xu} \mathrm{F}$, Porcher $\mathrm{R}$, et al. Pneumocystis jirovecii pneumonia in renal transplant recipients oc - curring after discontinuation of prophylaxis: a case - control study. Clin Microbiol Infect. 2010; 16(9): 13757. doi: 10.1111/j.1469-0691.2009.03143.x.

4. Fishman JA, Gans H, AST Infectious Diseases Community of Practice. Pneumocystis jiroveci in solid organ transplantation: Guidelines from the American Society of Transplantation Infectious Diseases Community of Practice. Clin Transplant. 2019, 33(9): e13587. doi: 10.1111/ctr.13587.

5. British Columbia transplant. CLINICAL GUIDELINES FOR KIDNEY TRANSPLANTATION.

http://www.transplant.bc.ca/Documents/Health Professionals/Clinical guidelines/Clinical Guidelines for Kidney Transplantation.pdf. Accessed June 25, 2019.

6. Kidney Disease: Improving Global Outcomes Transplant Work Group. KDIGO clinical practice guideline for the care of kidney transplant recipients. Am J Transplant. 2009; 9(Suppl 3): S1-155. doi: 10.1111/j.16006143.2009.02834.x.

7. Cincinnati Children's Hospital Medical Center. Antimicrobial prophylaxis for pneumocystis jiroveci pneumonia (PCP) after solid organ transplantation

(SOT).https://www.cincinnatichildrens.org//media/cincinnatichildrens/home/service/j/ander son-center/evidence-

basedcare/recommendations/type/antimicrobial prophylaxis best 206. Accessed October 21, 2019.

8. European best practice guidelines for renal transplantation. Section IV: Long-term management of the transplant recipient. IV.7.1 Late infections. Pneumocystis carinii pneumonia. 
Nephrol Dial Transplant. 2002; 17(Suppl 4): 369. PMID: 12091642.

9. Baker RJ, Mark PB, Patel RK, et al. Renal association clinical practice guideline in postoperative care in the kidney transplant recipient. BMC Nephrol. 2017; 18(1): 174. doi: 10.1186/s12882-017-0553-2.

10. Hughes WT, Rivera GK, Schell MJ, et al. Successful intermittent chemoprophylaxis for Pneumocystis carinii pneumonitis. N Engl J Med. 1987; 316(26): 1627-32. doi: 10.1056/NEJM198706253162604.

11. Olsen SL, Renlund DG, O'Connell JB, et al. Prevention of Pneumocystis carinii pneumonia in cardiac transplant recipients by trimethoprim sulfameth-oxazole. Transplantation. 1993; 56(2): 359-62. doi: 10.1097/00007890-19930800000021.

12. Rossi MR, Banfi P, Cappuccilli M, et al. Prospective randomized comparison of two prophylactic regimens with trimethoprimsulfamethoxazole in leuke-mic children: a two year study. Eur J Cancer Clin Oncol. 1987; 23(11): $1679-82$. doi: 10.1016/02775379(87)90449-4.

13. Bamoulid J, Staeck O, Halleck F, et al. The need for minimization strategies: current problems of immunosuppression. Transpl Int. 2015; 28: 891900. doi: 10.1016/0277-5379(87)90449-4.

14. Iriart X, Challan Belval T, Fillaux J, et al. Risk factors of Pneumocystis pneumonia in solid organ recipients in the era of the common use of posttransplantation prophylaxis. Am J Transplant. 2015; 15(1): 190-9. doi: 10.1111/ajt.12947.

15. de Boer MG, Kroon FP, le Cessie S, et al. Risk factors for Pneumocystis jirovecii pneumonia in kidney transplant recipients and appraisal of strategies for selective use of chemopro phylaxis. Transpl Infect Dis. 2011; 13(6): 55969. doi: 10.1111/j.1399-3062.2011.00645.x.

16. Borstnar S, Lindic J, Tomazic J, et al. Pneumocystis jirovecii pneumonia in renal transplant recipients: a national center experience. Transplant Proc. 2013; 45(4): 16147. doi: 10.1016/j.transproceed.2013.02.107.
17. Hosseini-Moghaddam SM, Shokoohi M, Singh $\mathrm{G}$, et al. A Multi-Center Case-Control Study of the Effect of Acute Rejection and Cytomegalovirus Infection on Pneumocystis Pneumonia (PCP) in Solid Organ Transplant Recipients. Clin Infect Dis. 2019; 68(8): 1320-6. doi: 10.1093/cid/ciy682.

18. Bayraktar A, Catma Y, Akyildiz A, et al. Infectious Complications of Induction Therapies in Kidney Transplantation. Ann Transplant. 2019; 24: 412-7. doi: 10.12659/AOT.915885.

19. Hill P, Cross NB, Barnett AN, et al. Polyclonal and monoclonal antibodies for induction therapy in kidney transplant recipients. Cochrane Database Syst Rev. 2017; 1(1): CD004759. doi: 10.1002/14651858.CD004759.pub2.

20. Chapman JR, Marriott DJ, Chen SC, et al. Posttransplant

Pneumocystis jirovecii pneumonia-a reemerged public health problem? Kidney Int. 2013; 84(2): 240-3. doi: 10.1038/ki.2013.212.

21. Wang EHZ, Partovi N, Levy RD, et al. Pneumocystis pneumonia in solid organ transplant recipients: not yet an infection of the past. Transpl Infect Dis. 2012; 14(5): 519-25. doi: 10.1111/j.1399-3062.2012.00740.x.

22. Goto N, Takahashi-Nakazato A, Futamura K, et al. Lifelong Prophylaxis With TrimethoprimSulfamethoxazole for Prevention of Outbreak of Pneumocystis jirovecii Pneumonia in Kidney Transplant Recipients. Transplant Direct. 2017; 3(5): e151. doi: 10.1097/TXD.0000000000000665.

23. Giullian JA, Cavanaugh K, Schaefer H. Lower risk of urinary tract infection with low-dose trimethoprim/sulfamethoxazole compared to dapsone prophylaxis in older renal transplant patients on a rapid steroid-withdrawal immunosuppression regimen. Clin Transplant. 2010 ; 24(5): 636-42. doi: 10.1111/j.13990012.2009.01129.x.

24. Mitsides N, Greenan K, Green D, et al. Complications and outcomes of trimethoprimsulphamethoxazole as chemoprophylaxis for pneumocystis pneumonia in renal transplant recipients. Nephrology (Carlton). 2014; 19(3): 157-63. doi: 10.1111/nep.12201. 